\title{
Correction to: A Structure-Behavior Coalescence Systems Modeling Approach for Service Systems Design
}

Yu-Chen Yang, Cheng-Ta Tsai, and William S. Chao

\section{Correction to:}

\section{Chapter "A Structure-Behavior Coalescence Method} for Human-Computer Interaction System Requirements Specification" in: F. F.-H. Nah and B. S. Xiao (Eds.):

HCI in Business, Government, and Organizations, LNCS 10923, https://doi.org/10.1007/978-3-319-91716-0_18

The title of the originally published chapter was identical with a chapter by the same authors published in 2016. To avoid confusion it has been corrected to "A StructureBehavior Coalescence Systems Modeling Approach for Service Systems Design". Additionally, typos were corrected in the legend of Figure 6 and in section 3.5. 